Sharif University of Technology
Scientia Iranica
Transactions E: Industrial Engineering
SCIENTIA
I RAN I C A

\title{
Solving a discrete congested multi-objective location problem by hybrid simulated annealing with customers' perspective
}

\author{
M. Ghobadi ${ }^{a}$, M. Seifbarghy ${ }^{a}$, R. Tavakoli-Moghadam ${ }^{\mathrm{b}}$ and D. Pishva $^{\mathrm{c}, *}$ \\ a. Faculty of Engineering, Alzahra University, Tehran, Iran. \\ b. Faculty of Industrial Engineering, University of Tehran, Tehran, Iran. \\ c. Faculty of Asia Pacific Studies, Ritsumeikan Asia Pacific University, Beppu, Japan.
}

Received 29 January 2014; received in revised form 1 October 2014; accepted 22 August 2015

\author{
KEYWORDS \\ Location-allocation; \\ Queuing; \\ Modeling; \\ Optimization; \\ VNS; \\ $\mathrm{SA}$; \\ Multi-objective.
}

\begin{abstract}
In the current competitive market, obtaining a greater share of the market requires consideration of the customers' preferences and meticulous demands. This study addresses this issue with a queuing model that uses multi-objective set covering constraints. It considers facilities as potential locations with the objective of covering all customers with a minimum number of facilities. The model is designed based on the assumption that customers can meet their needs by a single facility. It also considers three objective functions, namely minimizing the total number of the assigned server, minimizing the total transportation and facility deployment costs, and maximizing the quality of service from the customers' point of view. The main constraint is that every center should have less than $b$ numbers of people in line with a probability of at least $\alpha$ upon the arrival of a new customer. The feasibility of the approach is demonstrated by several examples which are designed and optimized by a proposed hybrid Simulated Annealing (SA) algorithm to evaluate the model's validity. Finally, the study compares the performance of the proposed algorithm with that of Variable Neighborhood Search (VNS) algorithm and concludes that it can arrive at an optimal solution in much less time than the VNS algorithm.

(C) 2016 Sharif University of Technology. All rights reserved.
\end{abstract}

\section{Introduction}

In recent years, due to the growing demand to reduce the transportation costs, attempts to model and optimize locations of commercial facilities have significantly increased. In general, these types of modeling are called location-allocation modeling. Location-allocation is about finding the best possible sites for one or more facilities by examining their relationship and associated

\footnotetext{
. Corresponding author. Tel.: +81 097778 1261;

Fax: +81 097r 78 1261

E-mail addresses: mrymghbd@gmail.com (M. Ghobadi);

m.seifbarghy@alzahra.ac.ir (M. Seifbarghy);

Tavakoli@ut.ac.ir (R. Tavakoli Moghadam);

dpishva@apu.ac.jp (D. Pishva)
}

constraints with existing and potential centers with the intention of optimizing them for a specific purpose. The optimization objective can be transportation cost reduction, providing fair services to the clients, gaining a greater share of the market, and so on. In locationallocation models, in addition to selecting the right places for facilities, careful consideration of customer demands and preferences can be a step forwards for the facilities' growth. Some important factors to consider are travel time and waiting time. Oftentimes, customers are quite annoyed when they are kept waiting for a long time for the service. This paper employs queuing techniques to review and optimize such factors in the modeling process. Considering that optimal location-allocation has to deal with many factors, the 
approach has been categorized based on issues it needs to deal with. Many studies have been carried out in the field and this section highlights some of the major ones.

A Set Covering Problem (SCP), which was first developed by Toregas et al. (1971), is one of the initial studies that aims to minimize the cost for a group of customers who receive services from multiple facilities [1]. Shanthikumar and Yao (1978) investigated server allocation models for the manufacturing site using a pre-defined queuing network that showed the location of work centers [2]. Hakimi (1983) introduced the competitive location model which followed the proximity rule in a network [3]. Revelle and Hogan (1988) proposed Probabilistic Location Set Covering Problem (PLSCP), which ensured that all demands were covered within a predetermined reliability [4]. Marianov and Revelle (1994) developed the PLSCP and proposed Queuing PLSCP (Q-PLSCP), which modeled each facility as a multi-server queuing system and optimized the waiting time by using server utilization ratio [5].

Marianov et al. (1999) studied the location problem in a competitive environment [6]. Marianov and Serra (2000) investigated the hierarchical location problem in a congested environment where all customers were initially referred to as a low-level server and elevated to a higher-level server on a need basis [7]. Marianov and Serra (2002) proposed a multi-server set covering problem with restriction on waiting time, wherein every center was restricted in such a way that probability of existing $b$ people in line upon arrival of a new customer could not be greater than $\alpha$ [8]. Shavandi and Mahlooji (2006) proposed a new mathematical model for location-allocation of emergency facilities such as hospitals, fire stations, and so on, by utilizing queue and fuzzy theory in the model [9]. Rajagopalan and Saydam (2009) proposed a new model for optimal location of ambulances with the objective of minimizing the travel distance while ensuring service support. Their approach utilizes hypercube queuing models to determine the probability of engaging any server and tabu search algorithm for maximizing the coverage [10]. Restrepo et al. (2009) extended the ambulance location modeling to an emergency system with the objective of allocating a certain number of ambulances to a set of sites in such a way that percentage of missing demand was minimized within a standard time limit [11].

Liu and $\mathrm{Xu}$ (2011) investigated a locationallocation problem in a fuzzy and random combinatorial environment, wherein a customer demand was expressed by a random combinatorial variable and transportation cost assumed by a fuzzy variable. They also proposed an integer linear programming model with genetic algorithm to solve the fuzzy locationallocation problem [12]. Chanta et al. (2011) focused on the performance of emergency service in the rural areas. Their main purpose was locating ambulances or mobile healthcare facilities in appropriate locations so as to balance availability of such services between urban and rural areas [13]. Arnaout (2011) used an ant colony algorithm to solve the Euclidean location-allocation problem with an undefined number of facilities and showed that the algorithm performed better than the genetic algorithm [14]. Drezner and Drezner (2011) handled a multi-server problem with the objective of minimizing the customer's travel time and waiting time. Their approach defined a number of facilities and assumed that each facility had an $M / M / K$ queue system. They used a descent algorithm, tabu search, and simulated annealing to solve the model [15]. $\mathrm{Li}$ et al. (2011) conducted an extensive literature review on relevant models and optimization methods for emergency facility location from the past few decades and proposed a new model for better handling of the situation [16].

Benneyan et al. (2012) provided single- and multi-period integer programming models to minimize procedure, travel, and set up costs simultaneously and increase network capacity based on the pertinent access constraints [17]. Rahmati et al. (2013) presented a multi-objective location model in a multiserver queuing network, in which the facility had $M / M / m$ queuing system. They used Multi-Objective Harmony Search (MOHS), a Pareto-based heuristic algorithm, to solve the problem. After validating the obtained results with Non-Dominated Sorting Genetic Algorithm (NSGA- $\|$ ) and Non-Dominated Ranking Genetic Algorithm (NRGA), they concluded that the proposed algorithm (MOHS) performed better than other algorithms in terms of computational time [18].

Mousavi et al. (2013) considered a capacitated location-allocation problem, in which customers' demands and their location were fuzzy and stochastic, respectively. Fuzzy programming was presented to model this problem and a hybrid intelligent algorithm was used to solve it. It should be noted that they used bivariate normal distribution for customers' location and fuzzy sets for their demands. They set the parameters of presented hybrid algorithm using Taguchi method. Lastly, they demonstrated numerical examples using this algorithm [19]. Adler et al. (2013) investigated the traffic police Routine Patrol Vehicle (RPV) assignment problem on an interurban road network through a series of integer linear programs. They developed four location-allocation models and applied them to a case study of the road network in northern Israel. The results of these models were compared to each other and in relation to the currently chosen locations and they presented a location-allocation configuration per RPV per shift with full call-for-service coverage whilst maximizing police presence and obviousness as a proxy 
for road safety [20]. Goswami (2014) investigated a discrete-time multiple-server queuing system in which inter-arrival and service time were assumed to be independent and geometrically distributed. The study also assumed that during an arrival, when all servers were busy, an arriving customer either entered the system with a probability of $b$ or moved to another facility with a probability of $1-b$. The study also showed that under special circumstances, the results could be generalized to those of continuous time systems [21].

This paper has adopted a probabilistic approach similar to that of the multi-server set covering problem, proposed by Marianov and Serra [8]. However, the presented model consists of three objective functions that:

1. Minimizes the total number of assigned servers;

2. Minimizes facility deployment cost and total transportation cost;

3. Maximizes the quality from the customers' point of view.

Each demand node must be allocated to a single facility located at a maximal distance from the demand node. The servers are located at only opened facilities and each facility should not have more than a predetermined number of waiting customers in line with a probability of at least $\alpha$ upon the arrival of a new customer. Pertinent notations and problem formulation for our approach are given in Section 2. In Section 3, we present solution algorithms including Simulated Annealing (SA), VNS, and hybrid SA. Sections 4 and 5 give some numerical examples by applying the proposed meta-heuristic algorithm to some hypothetical problems, presenting the associated results and carrying out some comparisons. Finally, Section 6 gives concluding remarks, identifies limitation of the findings, and provides suggestions for future research.

\section{Notations and problem formulation}

This section introduces mathematical notations for the objective functions and associated constraints, highlights underlying assumptions, formulates the necessary mathematical models, and briefly explains the model.

\subsection{Mathematical notations}

- $H_{j}\left(r_{j}, s_{j}\right)$ : Coordinate of the $j$ th potential location of deployment facility where $j=1,2, \cdots, n$;

- $P_{i}\left(a_{i}, b_{i}\right)$ : Coordinate of the $i$ th demand point (customer) where $i=1,2, \cdots, m$;

- $q_{j}$ : Quality of the $j$ th potential location in order to locate a facility;
- $F_{j}$ : Fixed deployment costs at the $j$ th potential location;

- $T$ : Transportation cost per unit of distance per demand (e.g., \$/number*m);

- $d(i ; j)$ : Direct distance between demand point $i$ and potential facility location $j$ is obtained as follows:

$$
d(i, j)=\sqrt{\left(r_{j}-a_{i}\right)^{2}+\left(s_{j}-b_{i}\right)^{2}} .
$$

- $C_{j}$ : Maximum number of servers which can be allocated to a potential location;

- $N_{i}$ : Set of potential locations which are located within a standard distance from demand point $i$;

- $B_{j}$ : Set of demand points which are located within a standard distance to potential location $j$;

- W: Maximum distance for demand points to be covered by a facility;

- $\rho_{\alpha u}$ : The minimal value of $\rho$ (i.e., facility workload) which makes Inequity (2) hold as an equality, provided that there are $u$ servers allocated at a given facility (as in [8]):

$$
\sum_{k=0}^{u-1} \frac{(u-k) u ! u^{f}}{k !} \frac{1}{\rho^{u+f+1-k}} \geq \frac{1}{1-\alpha} .
$$

Assuming that there are no more than $f$ people in line with a probability of at least $\alpha$ upon the arrival of a new customer in the given queuing system.

- $\rho_{\alpha u j}$ : The value of $\rho_{\alpha u}$ for facility $j$;

- $\lambda_{i}$ : Demand rate at demand node $i$;

- $\mu_{j}$ : Service rate of a facility at potential location $j$;

- $x_{i j}, y_{j u}$ : The decision variables are $x_{i j}$ and $z_{j u}$, wherein:

$x_{i j}= \begin{cases}1 & \begin{array}{l}\text { if customer } i \text { is assigned to facility } \\ \text { located at } j\end{array} \\ 0 & \text { otherwise }\end{cases}$

$$
y_{j u}= \begin{cases}1 & \begin{array}{l}
\text { if at least } u \text { servers are allocated at } \\
\text { potential location } j
\end{array} \\
0 & \text { otherwise }\end{cases}
$$

\subsection{Main assumptions}

We considered the following common assumptions in the model. Such assumptions are applied in many discrete congested facility location problems (e.g. $[7,8]$ ).

- Each facility utilizes $M / M / k_{j}$ queue system;

- Coverage area is defined for each facility;

- Number of servers at each facility is undefined; however, there is an upper bound for each facility; 
- Nature of the problem is discrete;

- Each demand (customer) can only use a single facility to fulfill its needs.

\subsection{Mathematical model}

By employing the aforementioned notations and assumptions, the associated mathematical model can be formulated as follows:

$$
\begin{aligned}
& \min Z_{1}=\sum_{j=1}^{n} \sum_{u=2}^{C_{j}} y_{j u}, \\
& \min Z_{2}=\sum_{j=1}^{n} F_{j} y_{j 1}+\sum_{j=1}^{n} \sum_{i=1}^{m} \lambda_{i} \times d(i, j) \times T \times x_{i j}, \\
& \max Z_{3}=\sum_{i=1}^{m} \sum_{j \in N_{i}} q_{j} x_{i j} .
\end{aligned}
$$

S.T.

$\sum_{j \in N_{i}} x_{i j}=1, \quad \forall i$

$y_{j u} \leq y_{j(u-1)}, \quad \forall j, \quad 2 \leq u \leq C_{j}$,

$\sum_{i=1}^{m} \sum_{j \in N_{i}} x_{i j} y_{j 1}=m$

$\sum_{i \in B_{j}} \lambda_{i} x_{i j} \leq \mu_{j}\left[y_{j 1} \rho_{\alpha 1 j}+\sum_{u=2}^{C_{j}} y_{j u}\left(\rho_{\alpha u j}-\rho_{\alpha(u-1) j}\right)\right]$,

$\forall j$,

$$
\begin{array}{ll}
x_{i j}=0 \quad \text { or } \quad 1, & \forall i, j, \\
y_{j u}=0 \quad \text { or } \quad 1, & \forall j, u .
\end{array}
$$

\subsection{Description of the model's statements}

Eq. (3) is for our first objective that minimizes the total number of servers. Eq. (4) is for our second objective that minimizes the facility deployment cost and total transportation cost. It is achieved by minimizing the deployment costs of facilities in potential locations and minimizing the demand cost at location $i$ by considering its distance and rate of demand. Eq. (5) achieves our third objective of maximizing the quality of service from the customers' point of view. Eq. (6) is a constraint that restricts each demand node to a single facility. Constraint (7) ensures location of servers to be at only open locations, and also ensures that $u-1$ server is allocated before allocating the $u$ th server to each facility. Eq. (8) is a constraint that ensures all demands are met by the facilities which have already been deployed in the desired location. Eq. (9) is a probabilistic constraint which limits every facility to have no more than $f$ people in line with a probability of at least $\alpha$ upon the arrival of a new customer. Eq. (10) is also a constraint that refers to the binary variables.

\section{Solution algorithms}

As mentioned earlier, this study uses both SA and VNS algorithms to solve the model. It then compares their respective analysis times and the quality of the outcomes in order to identify the superior algorithm. This section discusses these algorithms and some important aspects in coding them.

\subsection{Variable neighborhood search algorithm}

The Variable Neighborhood Search (VNS) algorithm is one of the new meta-heuristic algorithms, which is based on systematic changes of the neighborhood structure. This algorithm searches for the optimum solution in combinatorial optimization problems. Unlike many other meta-heuristic algorithms, this algorithm is quite simple and requires fewer parameters to be tuned. Achieving high-quality solutions in a reasonable period of time and the simplicity of this method indicate the efficiency of the algorithm. The VNS algorithm used in this study is derived from the basic case presented in [22] by Hansen and Mladenovic. The pseudo-code is shown in Figure 1.

The notion of VNS algorithm is based on the neighborhood structure changes, which prevents trapping into the localized optimization. As the problem and solution expand, the probability of trapping into a local minimum increases, hence the first step in the VNS algorithm is defining a neighborhood structure that generates a neighborhood solution. Furthermore, since VNS was designed for approximating solutions of discrete and continuous optimization problems, it can be used for solving linear program problems, integer program problems, mixed integer program problems, nonlinear program problems, etc.

\subsection{Simulated Annealing algorithm}

Simulated Annealing (SA) algorithm is a local search algorithm which is not trapped into the local optimum.

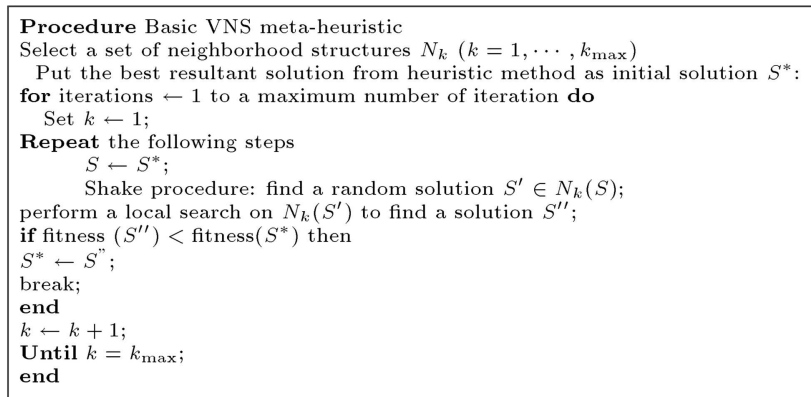

Figure 1. VNS pseudo-code. 


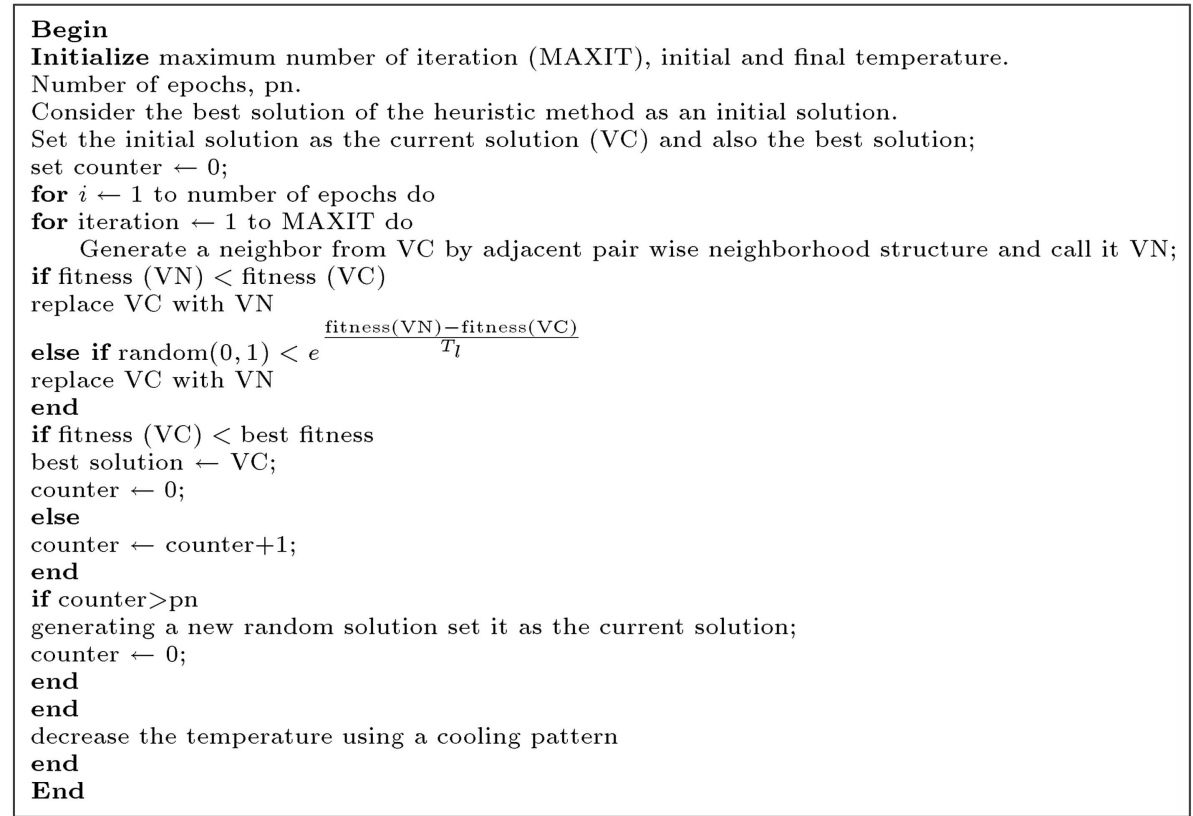

Figure 2. SA pseudo-code.

Its easy usage, convergence, and special movement to avoid being trapped into the local optimum are some of the advantages of this algorithm [23]. The pseudo-code is shown in Figure 2. The basic idea behind SA is from cooling process of metals, which was first suggested by Metropolis et al. [24] and optimized by Kirkpatricket et al. [25]. Despite generating a near-optimal solution, its outcome does not depend on the initial solution. Furthermore, even though it is an iterative algorithm, it does not have the common disadvantages of iterative methods as its upper limit execution time can also be specified. The basic idea originates in decreasing temperature of metals from an initial value of $T_{0}$ to a desired final value of $T_{f}$ in $N$ required iterations, which is called Epoch. The cooling pattern used here is given in Eq. (11), where Epoch is current number of iterations and $r$ is a constant number between 0 and 1 .

$$
T_{1}=T_{0}-\text { Epoch } \times r .
$$

SA has attracted significant attention as a suitable technique for optimization problems of large scale. The method has also been used successfully for designing complex integrated circuits and combinatorial minimization. Simulated annealing methods are also used for spaces with continuous control parameters. The SA algorithm presented in this paper includes some distinct features. First of all, it produces a random solution when the pre-determined number $(p n)$ does not yield the best outcome. Secondly, several neighborhood structures are generated and selected randomly by the algorithm in each iteration. The main advantage of the SA algorithm, compared to VNS, is its speedy response. In general, the VNS algorithm provides an optimal solution when its number of iterations leans towards infinity. In the SA algorithm, however, an optimal result is generated during a fixed number of iterations.

\subsection{Hybrid $S A$ algorithm}

In the proposed SA algorithm, the positive attributes of both SA and VNS algorithms are used simultaneously. Unlike the VNS algorithm, which uses several neighborhood structures, the SA algorithm considers only one neighborhood structure. In the proposed algorithm, one of the neighborhood structures is selected randomly and a neighbor is generated from the current solution. This procedure not only reduces the chances of obtaining repetitive answers, but also reduces the probability of trapping into the local optimum. Furthermore, in addition to defining the stop criteria for the algorithm, the convergence condition is also defined. Under this condition, a big number is assumed for the outer loop (i.e., Epoch) and if the problem does not improve after a certain number of iterations, it is assumed converged and the improvement process ends [26]. The general outline of the given meta-heuristic is shown in Figure 3.

- The objective function: The objective functions can be easily coded without requiring guide or competitive functions. However, the model is a multi-objective model and its objective functions are completely incompatible. When dealing with multiobjective modeling, one of the main challenges is to obtain a solution that optimizes all of its objective functions. Oftentimes, obtaining such an optimal solution becomes impossible because of the existence of conflicts of interest among the objective functions. This study uses the $L_{p}$-metric method (with $p=$ $\infty$ ) in which the objective function is minimizing 


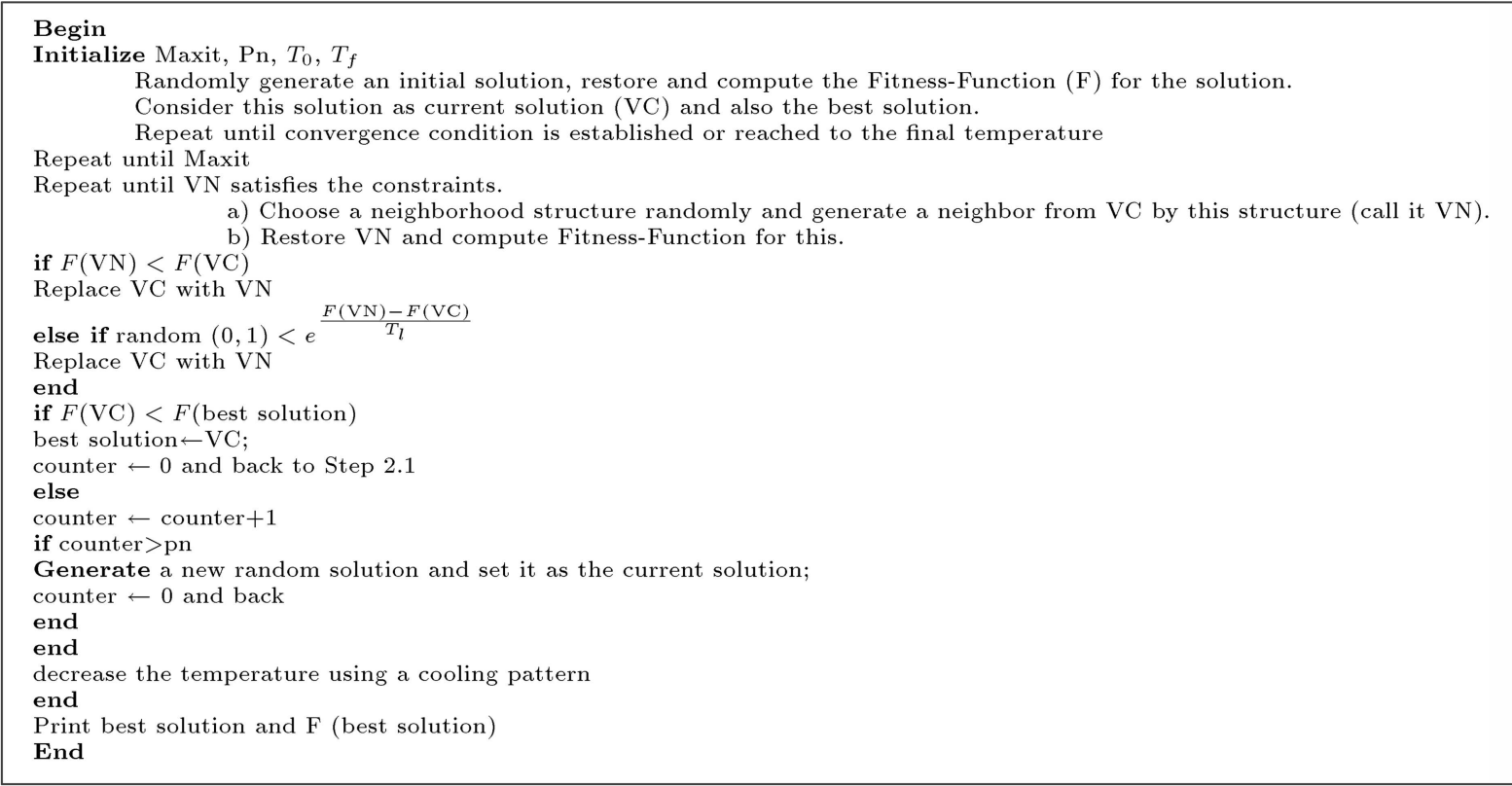

Figure 3. Hybrid SA pseudo-code.

deviations of the existing objective functions from their optimal values as indicated in Eq. (12). In other words, when $p$ is infinity, to minimize $L_{P}$ we need to minimize $Z$ and the final mathematical model can be denoted by Eqs. (12) and (13) subject to the initial constraints of the original model as indicated earlier in Constraints (6)-(10).

$$
\begin{aligned}
\min Z=\max \{ & \gamma_{1}\left(\frac{Z_{1}-Z_{1}^{*}}{Z_{1}^{*}}\right), \gamma_{2}\left(\frac{Z_{2}-Z_{2}^{*}}{Z_{2}^{*}}\right), \\
& \left.\gamma_{3}\left(\frac{Z_{3}-Z_{3}^{*}}{Z_{3}^{*}}\right)\right\}=\beta
\end{aligned}
$$

\section{S.T.}

$$
\beta \geq \gamma_{j}\left(\frac{Z_{j}-Z_{j}^{*}}{Z_{j}^{*}}\right) \forall j
$$

assuming that:

$$
\gamma_{1}+\gamma_{2}+\gamma_{3}=1
$$

- Solution representation: As iterative metaheuristic algorithms require a structure for solution representation, this study purposes binary encoding, wherein each solution is represented by a string of $0 \mathrm{~s}$ and $1 \mathrm{~s}$. This is rather a common approach and the following matrices are an example of a numerical solution for a scenario, in which there are three customers, three facilities, and up to 3 servers for each facility:

$$
X_{i j}=\left[\begin{array}{lll}
1 & 0 & 0 \\
1 & 0 & 0 \\
0 & 0 & 1
\end{array}\right], \quad Y_{j u}=\left[\begin{array}{lll}
1 & 1 & 0 \\
0 & 0 & 0 \\
1 & 0 & 0
\end{array}\right]
$$

$0 \mathrm{~s}$ and $1 \mathrm{~s}$ in matrix $X_{i j}$ indicate how to allocate customers to facilities (Customer 1 and Customer 2 are assigned to the facility at potential Location 1 and Customer 3 to the facility at potential Location 3). Numbers in matrix $Y_{j u}$ show how to allocate servers to facilities (i.e., two servers are assigned to the first facility and one server to the third one). In this solution, since the second facility has not yet been established, no server (i.e., the second row of the matrix $Y_{j u}$ ) and customer (i.e., the second column of the matrix $X_{i j}$ ) are assigned to it.

- Constraints: The proposed model contains certain constraints that need to be defined so as to code its associated meta-heuristic algorithm. The model includes linear, nonlinear, equality, and inequality constraints. The strategy employed in this study is a "reject strategy", which has a simple approach of considering feasible solutions and declining infeasible ones. This strategy has been used in dealing with Constraint (9). The model also includes other constraints (i.e., Constraints (6), (7), (8), and (10)), which can be included in the solution structure. Hence, we can generate feasible solutions for the problem utilizing the mentioned strategies.

\section{Numerical examples}

In order to clearly demonstrate convergence of the model and its effectiveness, and to objectively compare results of the two algorithms, several examples are designed and solved using the proposed hybrid SA and VNS algorithms. The solution algorithms are written in Matlab software 7.8.0 and tested on an Intel 
Core i5 Computer having a CPU of $2.4 \mathrm{GHz}$ and a RAM of 4 GB. For effective presentation purposes, after showing comprehensive solution for one sample example, the method is generalized and applied to other examples (1-6).

The following are considered for the sample example:

- Set of customers including 30 points;

- Set of potential locations for deployment of facilities including 10 points;

- Transportation cost per distance unit per demand unit is assumed to be 1 ;

- Maximum distance for a demand point to be covered by a facility is set to 5 ;

- Maximum number of people in queue on the arrival of each customer, with probability of 0.9 , is 5 .

Tables 1 and 2 indicate a comprehensive list of the relevant data. In designing the examples, we have considered existence of feasible area for each scenario.

Parameters of the algorithms have to be tuned prior to being applied to the examples. This means choosing the best possible values for parameters for the purpose of achieving optimal performance (the best possible performance of algorithm). These parameters may have great impact on the efficiency and effectiveness of the algorithm. In general, providing optimum values for the parameters of a meta-heuristic algorithm is not possible and should be examined separately for each numerical example. There are various strategies to tune the parameters and this research uses sequential strategy.

In the sequential strategy approach, each parameter is investigated individually and their optimum values are determined experimentally. As no interactive effects of parameters on each other can be determined in this approach, Design Of Experiments (DOE) is used to address this issue. In this way, the optimality of the parameters can be determined by considering the interaction between them.

The hybrid SA parameters, including MAXIT, $T_{0}$, and $p n$, need to be tuned. In the VNS algorithm, because there is just one parameter, the trial and error method can be adopted and it is used to compute optimal value of the parameter. Table 3 shows the tuned values of these parameters for the sample example. Subsequently, the problem is solved with the Lp-metric method that requires the optimal value of each function separately. The optimal value and solution time of each function are shown in Table 4.

In this example, the convergence condition for the LP is considered passing 30 successive iterations without any change in the best objective function value. Table 5 shows the achieved outcomes from solving

Table 3. Optimal values of the hybrid SA algorithm parameters for the sample example.

\begin{tabular}{ccc}
\hline Parameter & Upper-lower & Optimum value \\
\hline Maxit & $100-500$ & $\mathbf{3 0 0}$ \\
$\boldsymbol{T}_{\mathbf{0}}$ & $1000-4000$ & $\mathbf{2 0 0 0}$ \\
$\boldsymbol{p n}$ & $10-20$ & $\mathbf{1 0}$ \\
\hline
\end{tabular}

Table 1. Relevant data of the potential locations for the sample example.

\begin{tabular}{ccccccccccc}
\hline Potential location & $\mathbf{1}$ & $\mathbf{2}$ & $\mathbf{3}$ & $\mathbf{4}$ & $\mathbf{5}$ & $\mathbf{6}$ & $\mathbf{7}$ & $\mathbf{8}$ & $\mathbf{9}$ & $\mathbf{1 0}$ \\
\hline $\boldsymbol{q}_{\boldsymbol{j}}$ & 2 & 4 & 1 & 3 & 5 & 3 & 2 & 5 & 4 & $\mathbf{3}$ \\
$\boldsymbol{F}_{\boldsymbol{j}}$ & 1000 & 1200 & 1300 & 1400 & 1500 & 1600 & 1700 & 1800 & 1900 & $\mathbf{1 8 0 0}$ \\
$\boldsymbol{C}_{\boldsymbol{j}}$ & 5 & 4 & 3 & 2 & 8 & 2 & 3 & 4 & 5 & $\mathbf{7}$ \\
$\boldsymbol{\mu}_{\boldsymbol{j}}$ & 4 & 6 & 5 & 10 & 3 & 9 & 6 & 5 & 4 & $\mathbf{3}$ \\
$\boldsymbol{H}_{\boldsymbol{j}}$ & $(4,6)$ & $(3,2)$ & $(1.5,4)$ & $(6,6)$ & $(5,1)$ & $(9,2)$ & $(6,3)$ & $(3,6)$ & $(1,8)$ & $\mathbf{( 5 , 2 )}$ \\
\hline
\end{tabular}

Table 2. Relevant data of demand points for the sample example.

\begin{tabular}{ccccccccccc}
\hline Demand point & $\mathbf{1}$ & $\mathbf{2}$ & $\mathbf{3}$ & $\mathbf{4}$ & $\mathbf{5}$ & $\mathbf{6}$ & $\mathbf{7}$ & $\mathbf{8}$ & $\mathbf{9}$ & $\mathbf{1 0}$ \\
\hline $\boldsymbol{\lambda}_{\boldsymbol{i}}$ & 2 & 3 & 6 & 9 & 7 & 5 & 4 & 3 & 8 & 8 \\
$\boldsymbol{P}_{\boldsymbol{i}}$ & $(1,2)$ & $(2,6)$ & $(3,7)$ & $(6,5)$ & $(2,3)$ & $(3,4)$ & $(1,7)$ & $(2,4)$ & $(3,5)$ & $(4,1)$ \\
\hline Demand point & $\mathbf{1 1}$ & $\mathbf{1 2}$ & $\mathbf{1 3}$ & $\mathbf{1 4}$ & $\mathbf{1 5}$ & $\mathbf{1 6}$ & $\mathbf{1 7}$ & $\mathbf{1 8}$ & $\mathbf{1 9}$ & $\mathbf{2 0}$ \\
\hline $\boldsymbol{\lambda}_{\boldsymbol{i}}$ & 6 & 4 & 5 & 9 & 10 & 3 & 2 & 5 & 8 & 4 \\
$\boldsymbol{P}_{\boldsymbol{i}}$ & $(2,2)$ & $(5,3)$ & $(4,6)$ & $(3,8)$ & $(2,1)$ & $(7,4)$ & $(6,9)$ & $(8,2)$ & $(10,6)$ & $(2,8)$ \\
\hline Demand point & $\mathbf{2 1}$ & $\mathbf{2 2}$ & $\mathbf{2 3}$ & $\mathbf{2 4}$ & $\mathbf{2 5}$ & $\mathbf{2 6}$ & $\mathbf{2 7}$ & $\mathbf{2 8}$ & $\mathbf{2 9}$ & $\mathbf{3 0}$ \\
\hline $\boldsymbol{\lambda}_{\boldsymbol{i}}$ & 6 & 3 & 2 & 3 & 7 & 5 & 7 & 2 & 1 & 10 \\
$\boldsymbol{P}_{\boldsymbol{i}}$ & $(3,3)$ & $(1,5)$ & $(4,7)$ & $(7,1)$ & $(4,9)$ & $(9,7)$ & $(5,3)$ & $(4,5)$ & $(2,8)$ & $(7,9)$ \\
\hline
\end{tabular}


Table 4. Optimum value and solution time of each function for the sample example.

\begin{tabular}{lcccccc}
\hline & \multicolumn{2}{c}{ Hybrid SA algorithm } & & \multicolumn{2}{c}{ VNS algorithm } \\
\cline { 2 - 3 } & $\begin{array}{c}\text { Optimum } \\
\text { value }\end{array}$ & $\begin{array}{c}\text { Solution } \\
\text { time (s) }\end{array}$ & & $\begin{array}{c}\text { Optimum } \\
\text { value }\end{array}$ & $\begin{array}{c}\text { Solution } \\
\text { time (s) }\end{array}$ \\
\hline First function (servers) & 18 & 2.50 & & 11 & $\mathbf{1 8 . 7 7}$ \\
Second function (cost) & 15311 & 2.46 & & 15310 & $\mathbf{1 9 . 2 0}$ \\
Third function (quality) & 97 & 2.48 & & 97 & $\mathbf{1 9 . 5 0}$ \\
\hline
\end{tabular}

Table 5. Achieved outcomes from solving the LP with different combinations of the weights for the sample example.

\begin{tabular}{|c|c|c|c|c|c|c|c|c|c|c|c|c|c|c|c|}
\hline \multirow[b]{2}{*}{$\gamma_{j}$} & \multicolumn{5}{|c|}{ Hybrid SA algorithm } & \multicolumn{5}{|c|}{ VNS algorithm } & \multicolumn{5}{|c|}{ Branch and bound } \\
\hline & $\begin{array}{l}\text { Optimum } \\
\text { value }\end{array}$ & $\begin{array}{c}\text { Solution } \\
\text { time } \\
(\mathrm{s})\end{array}$ & $Z_{1}$ & $Z_{2}$ & $Z_{3}$ & $\begin{array}{l}\text { Optimum } \\
\text { value }\end{array}$ & $\begin{array}{c}\text { Solution } \\
\text { time } \\
(\mathrm{s})\end{array}$ & $Z_{1}$ & $Z_{2}$ & $Z_{3}$ & $\begin{array}{l}\text { Optimum } \\
\text { value }\end{array}$ & $\begin{array}{c}\text { Solution } \\
\text { time } \\
(\mathrm{s})\end{array}$ & $Z_{1}$ & $Z_{2}$ & $Z_{3}$ \\
\hline $0.6-0.1-0.3$ & 0.0096 & 4.03 & 22 & 15500 & 51 & & 3.71 & 18 & 15441 & 34 & 5 & 8 & 18 & 15440 & 51 \\
\hline $0.1-0.3-0.6$ & 0.0286 & 5.41 & 23 & 15451 & 55 & $1.6 \mathrm{e}-3$ & 4.86 & 19 & 15392 & 42 & $3,001 \mathrm{e}-5$ & 11 & 19 & 15392 & 55 \\
\hline $0.3-0.6-0.1$ & 0.0572 & 4.76 & 20 & 15604 & 51 & $7.594 \mathrm{e}-4$ & 4.72 & 19 & 15329 & 34 & $3,023 \mathrm{e}-5$ & 9 & 19 & 15317 & 51 \\
\hline
\end{tabular}

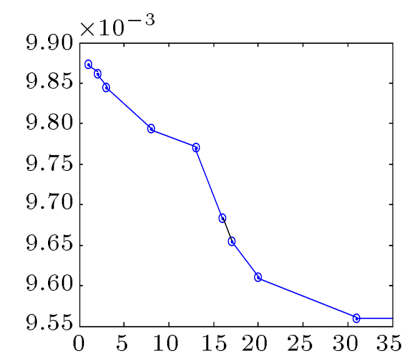

(a)

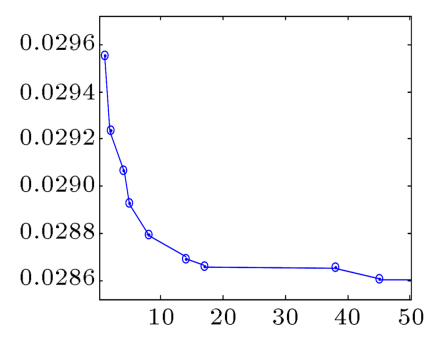

(b)

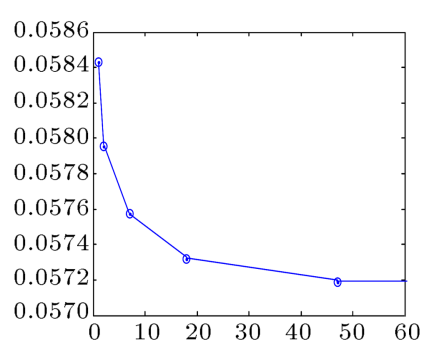

(c)

Figure 4. Improvement process for the proposed hybrid SA algorithm regarding the sample example with $\gamma$ equal to (a) $0.3,0.1$, and 0.6 , (b) 0.6, 0.3, and 0.1, and (c) 0.1, 0.6, and 0.3 .

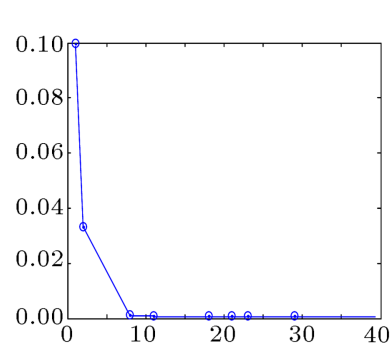

(a)

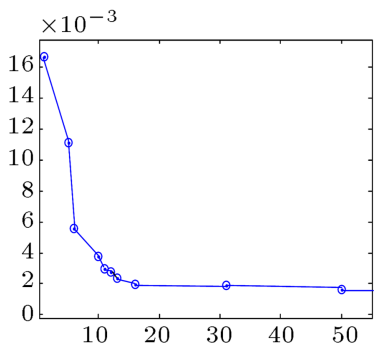

(b)

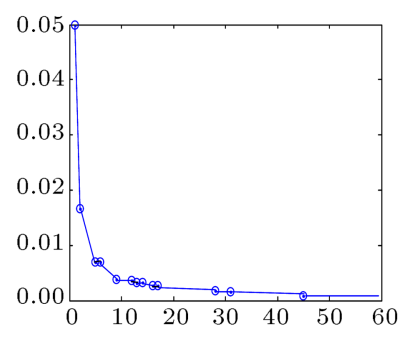

(c)

Figure 5. Improvement process for the VNS algorithm regarding the sample example with $\gamma$ equal to (a) $0.3,0.1$, and 0.6 , (b) $0.6,0.3$, and 0.1 , and (c) $0.1,0.6$, and 0.3 .

the LP with different combinations of the weights for the sample example. Also, in this table, the achieved outcomes from solving the LP with branch and bound method are shown to compare the proposed method with an exact method. It can be seen that outcomes of these two methods are very close to those of the optimal solutions. Figures 4 and 5 show the improvement process of these six cases wherein the horizontal axis represents the number of iterations, in which the algorithm shows improvement and the vertical axis represents the best value of the objective function. Now that convergence of the two algorithms is demonstrated, we need to calculate an index called RPI for the purpose of comparing the two algorithms. The next section discusses a general process of calculating the index and shows the associated results for the above-mentioned examples.

\section{RPI method for comparing the algorithms}

As mentioned earlier, the RPI is used to compare the efficiency of algorithms in solving problems. The 
general formula of the index is represented in Eq. (15):

$$
\mathrm{RPI}=\left|\frac{\text { best }- \text { objective value }}{\text { best }- \text { worst }}\right| .
$$

The following steps are used to calculate the RPI for efficiency comparison:

- Each algorithm is run five times for a numerical example of the problem;

- The objective function values are acquired for each algorithm during each run;

- The best and the worst objective function values are identified;

- RPI is calculated for each objective function in each run;

- The average value of RPI $(\bar{R})$ is calculated for each algorithm.

As the process shows in Table 6, the index for the sample example is calculated. All the above steps are repeated for the given six numerical examples, the results of which are summarized in Table 7 . The comparative statistical tests are used to compare $\bar{R}$ s. In this study, the 2 -sample $t$-test with a confidence level

Table 6. Results of $\bar{R}$ for the sample example.

\begin{tabular}{|c|c|c|c|c|}
\hline \multirow[b]{2}{*}{$\gamma_{j}$} & \multicolumn{2}{|c|}{ SA } & \multicolumn{2}{|c|}{ VNS } \\
\hline & $\begin{array}{c}\text { LP } \\
\text { objective } \\
\text { value }\end{array}$ & $\begin{array}{c}\text { LP } \\
\text { solution } \\
\text { time (S) }\end{array}$ & $\begin{array}{c}\text { LP } \\
\text { objective } \\
\text { value }\end{array}$ & $\begin{array}{c}\text { LP } \\
\text { solution } \\
\text { time }(\mathrm{S})\end{array}$ \\
\hline \multirow{5}{*}{ 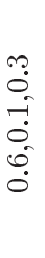 } & 0.0096 & 2.3 & $1.2 \mathrm{e}-4$ & 4.6 \\
\hline & 0.0096 & 2.9 & $6.3 \mathrm{e}-4$ & 3.5 \\
\hline & 0.0097 & 2.3 & $5.7 \mathrm{e}-4$ & 2.7 \\
\hline & 0.0095 & 4.7 & $6.9 \mathrm{e}-4$ & 2.8 \\
\hline & 0.0095 & 4.9 & $0.0 \mathrm{e}-0$ & 7.0 \\
\hline $\bar{R}$ & 0.4000 & 0.43 & $5.82 \mathrm{e}-1$ & 0.32 \\
\hline \multirow{5}{*}{$\begin{array}{l}0 \\
0 \\
0 \\
0 \\
-1 \\
0\end{array}$} & 0.0282 & 2.2 & $1.1 \mathrm{e}-4$ & 5.4 \\
\hline & 0.0286 & 2.7 & $2.0 \mathrm{e}-3$ & 2.8 \\
\hline & 0.0283 & 5.2 & $5.8 \mathrm{e}-4$ & 4.7 \\
\hline & 0.0285 & 4.5 & $1.4 \mathrm{e}-3$ & 4.1 \\
\hline & 0.0285 & 8.1 & $1.7 \mathrm{e}-3$ & 4.4 \\
\hline $\bar{R}$ & 0.5500 & 0.40 & $5.54 \mathrm{e}-1$ & 0.57 \\
\hline \multirow{5}{*}{$\begin{array}{l}-1 \\
\vdots \\
0 \\
0 \\
0 \\
0 \\
ٌ \\
0\end{array}$} & 0.0571 & 2.2 & $0.0 \mathrm{e}-0$ & 4.8 \\
\hline & 0.0575 & 3.5 & $3.2 \mathrm{e}-3$ & 7.3 \\
\hline & 0.0574 & 4.0 & $1.0 \mathrm{e}-3$ & 6.8 \\
\hline & 0.0574 & 3.2 & $4.9 \mathrm{e}-3$ & 6.9 \\
\hline & 0.0573 & 2.6 & $1.2 \mathrm{e}-3$ & 3.5 \\
\hline $\bar{R}$ & 0.6000 & 0.50 & $4.2 \mathrm{e}-3$ & 0.62 \\
\hline
\end{tabular}

of 0.95 is used and the following assumptions are also tested. The first investigated hypothesis is to identify any differences between the qualities of the obtained solution and the two algorithms.

Thus, $H_{0}$ and $H_{1}$ are as follows:

$$
H_{0}: \mu_{\mathrm{SA}} \geq \mu_{\mathrm{VNS}}, \quad H_{1}: \mu_{\mathrm{SA}}<\mu_{\mathrm{VNS}} .
$$

Hypothesis $1\left(H_{1}\right)$ means that SA algorithm has better performance than VNS. This is because the mean objective function value (as the solution quality) of SA was assumed to be less than or equal to that of VNS. The $P$-Value turns out to be equal to 0.308 , which implies that with a $95 \%$ confidence, $H_{1}$ cannot be accepted.

Aside from the quality of the solutions obtained from an algorithm, time to achieve the optimal solution is also an important factor in selecting an algorithm. Therefore, the second hypothesis is defined by:

$$
H_{0}: \mu_{t \mathrm{SA}} \geq \mu_{t \mathrm{VNS}}, \quad H_{1}: \mu_{t \mathrm{SA}}<\mu_{t \mathrm{VNS}} .
$$

Hypothesis $1\left(H_{1}\right)$ means that $\mathrm{SA}$ algorithm reaches the corresponding solution faster than VNS. This is again because the mean time for SA was assumed to be less than or equal to that for VNS. As $P$-Value turns out to be equal to 0.026 , it indicates that by $95 \%$ confidence, $H_{1}$ can be accepted.

\section{Conclusion}

In this paper, several potential locations were considered and we aimed at locating a number of facilities at those locations, each equipped with some servers. The total number of servers was considered unknown, but the maximum number of servers that could be allocated to each facility was specified and when deploying a location, at least one server was allocated to it. We proposed a model based on the customers' perspective and optimized its three objective functions of:

1. Minimizing the total number of assigned servers;

2. Minimizing the total transportation and the facility deployment costs;

3. Maximizing the quality of service from the customers' point of view, in order to attain our objectives.

It was shown that the hybrid SA algorithm attains near-optimal solutions more efficiently and sequential strategy was used for tuning of its parameters. Some numerical examples, which were designed to evaluate the algorithm's performance, were demonstrated. Finally, the two algorithms of SA and VNS were compared by the RPI method in order to identify the best performing algorithm. The results indicated that there was no significant difference between the qualities 
Table 7. Results of $\bar{R}$ for examples 1-6.

\begin{tabular}{|c|c|c|c|c|c|c|c|c|c|c|c|}
\hline \multirow[b]{2}{*}{ 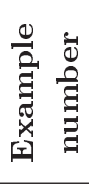 } & \multirow[b]{2}{*}{$\gamma_{j}$} & \multicolumn{2}{|c|}{ SA } & \multicolumn{2}{|c|}{ VNS } & \multirow[b]{2}{*}{ 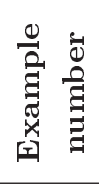 } & \multirow[b]{2}{*}{$\gamma_{j}$} & \multicolumn{2}{|c|}{ SA } & \multicolumn{2}{|c|}{ VNS } \\
\hline & & $\begin{array}{c}\text { LP } \\
\text { objective } \\
\text { value } \\
\end{array}$ & $\begin{array}{c}\text { LP } \\
\text { solution } \\
\text { time (s) } \\
\end{array}$ & $\begin{array}{c}\text { LP } \\
\text { objective } \\
\text { value } \\
\end{array}$ & $\begin{array}{c}\text { LP } \\
\text { solution } \\
\text { time (s) }\end{array}$ & & & $\begin{array}{c}\text { LP } \\
\text { objective } \\
\text { value } \\
\end{array}$ & $\begin{array}{c}\text { LP } \\
\text { solution } \\
\text { time (s) }\end{array}$ & $\begin{array}{c}\text { LP } \\
\text { objective } \\
\text { value } \\
\end{array}$ & $\begin{array}{c}\text { LP } \\
\text { solution } \\
\text { time (s) }\end{array}$ \\
\hline \multirow{18}{*}{ 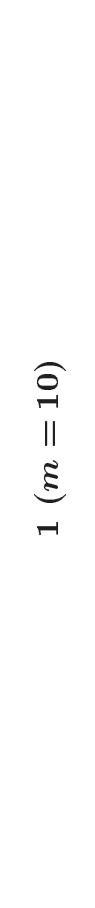 } & \multirow{5}{*}{$\begin{array}{l}3 \\
0 \\
-1 \\
0 \\
0 \\
0 \\
0\end{array}$} & 0.0046 & 3.21 & $7.9 \mathrm{e}-4$ & 0.93 & \multirow{18}{*}{$\begin{array}{l}\text { Oิ } \\
10 \\
11 \\
\text { ह } \\
\text { N }\end{array}$} & \multirow{5}{*}{$\begin{array}{l}r \\
0 \\
-1 \\
0 \\
0 \\
0 \\
0\end{array}$} & 0.0482 & 41.3 & $1.8 \mathrm{e}-3$ & 49.3 \\
\hline & & 0.0044 & 3.10 & $7.8 \mathrm{e}-4$ & 0.96 & & & 0.0482 & 27.2 & $1.9 \mathrm{e}-3$ & 48.9 \\
\hline & & 0.0044 & 2.89 & $7.9 \mathrm{e}-4$ & 0.74 & & & 0.0481 & 20.7 & $2.0 \mathrm{e}-3$ & 19.5 \\
\hline & & 0.0042 & 2.81 & $7.9 \mathrm{e}-4$ & 0.72 & & & 0.0482 & 23.9 & $2.0 \mathrm{e}-3$ & 28.16 \\
\hline & & 0.0044 & 2.97 & $7.8 \mathrm{e}-4$ & 0.71 & & & 0.0482 & 27.9 & $2.0 \mathrm{e}-3$ & 32.6 \\
\hline & $\overline{\boldsymbol{R}}$ & 0.5 & 0.465 & $6.0 \mathrm{e}-1$ & 0.463 & & $\overline{\boldsymbol{R}}$ & 0.8 & 0.364 & $7.0 \mathrm{e}-1$ & 0.542 \\
\hline & \multirow{5}{*}{$\begin{array}{l}0 \\
0 \\
0 \\
0 \\
0 \\
-1 \\
0\end{array}$} & 0.0137 & 5.84 & $2.4 \mathrm{e}-3$ & 0.73 & & \multirow{5}{*}{ 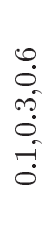 } & 0.1445 & 26.9 & $5.9 \mathrm{e}-3$ & 28.6 \\
\hline & & 0.0131 & 5.62 & $2.4 \mathrm{e}-3$ & 0.87 & & & 0.1445 & 42.6 & $5.9 \mathrm{e}-3$ & 21.3 \\
\hline & & 0.0131 & 5.97 & $2.3 \mathrm{e}-3$ & 0.89 & & & 0.1447 & 38.6 & $6.1 \mathrm{e}-3$ & 40.3 \\
\hline & & 0.0132 & 6.26 & $2.5 \mathrm{e}-3$ & 0.76 & & & 0.1446 & 26.5 & $6.2 \mathrm{e}-3$ & 18.4 \\
\hline & & 0.0131 & 5.93 & $2.3 \mathrm{e}-3$ & 0.78 & & & 0.1444 & 27.2 & $5.7 \mathrm{e}-3$ & 50.2 \\
\hline & $\overline{\boldsymbol{R}}$ & 0.233 & 0.475 & $4.0 \mathrm{e}-1$ & 0.475 & & $\overline{\boldsymbol{R}}$ & 0.466 & 0.363 & $5.2 \mathrm{e}-1$ & 0.42 \\
\hline & \multirow{5}{*}{$\begin{array}{l}-1 \\
0 \\
0 \\
0 \\
0 \\
0 \\
0\end{array}$} & 0.0262 & 2.84 & $4.9 \mathrm{e}-3$ & 0.71 & & \multirow{5}{*}{$\begin{array}{l}\breve{1} \\
0 \\
0 \\
0 \\
0 \\
0 \\
0\end{array}$} & 0.2893 & 26.6 & $1.09 \mathrm{e}-2$ & 31.4 \\
\hline & & 0.0262 & 2.77 & $4.8 \mathrm{e}-3$ & 0.76 & & & 0.2888 & 27.2 & $1.06 \mathrm{e}-2$ & 51.9 \\
\hline & & 0.0263 & 2.87 & $4.8 \mathrm{e}-3$ & 0.71 & & & 0.2887 & 25.4 & $1.12 \mathrm{e}-2$ & 54.4 \\
\hline & & 0.0263 & 2.83 & $4.9 \mathrm{e}-3$ & 0.95 & & & 0.2892 & 30.4 & $1.19 \mathrm{e}-2$ & 22.3 \\
\hline & & 0.0262 & 2.79 & $4.7 \mathrm{e}-3$ & 0.71 & & & 0.2892 & 56.4 & $1.16 \mathrm{e}-2$ & 28.9 \\
\hline & $\bar{R}$ & 0.4 & 0.5 & $6.0 \mathrm{e}-1$ & 0.241 & & $\overline{\boldsymbol{R}}$ & 0.566 & 0.251 & $4.92 \mathrm{e}-1$ & 0.482 \\
\hline \multirow{18}{*}{ 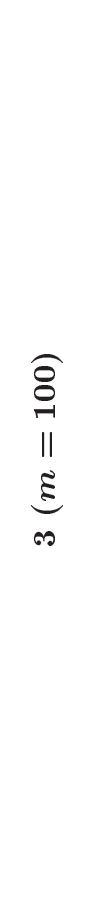 } & \multirow{5}{*}{$\begin{array}{l}0 \\
0 \\
-1 \\
0 \\
0 \\
0 \\
0\end{array}$} & 0.0069 & 148.7 & $7.7 \mathrm{e}-3$ & 125 & \multirow{18}{*}{ 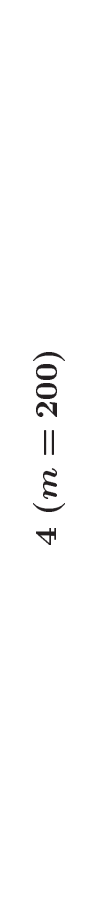 } & \multirow{5}{*}{$\begin{array}{l}3 \\
0 \\
-1 \\
0 \\
0 \\
0 \\
0\end{array}$} & 0.0071 & 2235.4 & $6.3 \mathrm{e}-3$ & 2010 \\
\hline & & 0.0069 & 282.7 & $6.4 \mathrm{e}-5$ & 307 & & & 0.0092 & 2594.1 & $8.6 \mathrm{e}-3$ & 1927.4 \\
\hline & & 0.0072 & 161.3 & $5.1 \mathrm{e}-5$ & 284 & & & 0.0068 & 2367.3 & $1.01 \mathrm{e}-2$ & 741.4 \\
\hline & & 0.0073 & 191 & $0.0 \mathrm{e}-0$ & 287 & & & 0.0101 & 2721.3 & $9.7 \mathrm{e}-3$ & 1991.7 \\
\hline & & 0.0072 & 160.7 & $1.54 \mathrm{e}-2$ & 137. & & & 0.0075 & 2714.9 & $1.08 \mathrm{e}-2$ & 606.3 \\
\hline & $\overline{\boldsymbol{R}}$ & 0.5 & 0.3 & $3.01 \mathrm{e}-1$ & 0.567 & & $\overline{\boldsymbol{R}}$ & 0.406 & 0.599 & $6.22 \mathrm{e}-1$ & 0.604 \\
\hline & \multirow{5}{*}{$\begin{array}{l}0 \\
0 \\
0 \\
0 \\
0 \\
-1 \\
0\end{array}$} & 0.0213 & 141.7 & $2.6 \mathrm{e}-3$ & 211 & & \multirow{5}{*}{$\begin{array}{l}0 \\
0 \\
0 \\
0 \\
0 \\
-1 \\
0\end{array}$} & 0.0018 & 2902.2 & $1.1 \mathrm{e}-3$ & 1219.3 \\
\hline & & 0.0212 & 174.5 & $9.3 \mathrm{e}-5$ & 363 & & & 0.0051 & 2941.3 & $2.8 \mathrm{e}-3$ & 817.4 \\
\hline & & 0.0212 & 236.4 & $3.8 \mathrm{e}-3$ & 181 & & & 0.0028 & 2851.4 & $1.2 \mathrm{e}-3$ & 645.77 \\
\hline & & 0.0212 & 256.1 & $1.3 \mathrm{e}-3$ & 222 & & & 0.0031 & 2512.8 & $3.1 \mathrm{e}-3$ & 935.8 \\
\hline & & 0.0214 & 180.8 & $3.8 \mathrm{e}-4$ & 112 & & & 0.0020 & 2638.4 & $6.0 \mathrm{e}-3$ & 614.2 \\
\hline & $\overline{\boldsymbol{R}}$ & 0.3 & 0.491 & $4.15 \mathrm{e}-1$ & 0.52 & & $\overline{\boldsymbol{R}}$ & 0.351 & 0.598 & $3.55 \mathrm{e}-1$ & 0.474 \\
\hline & & 0.0408 & 286.5 & $0.0 \mathrm{e}-0$ & 382 & & & 0.0053 & 2042.1 & $1.8 \mathrm{e}-3$ & 989.8 \\
\hline & -1. & 0.0427 & 252.9 & $6.2 \mathrm{e}-4$ & 599 & & $\overrightarrow{0}$ & 0.0067 & 2430.3 & $3.8 \mathrm{e}-3$ & 724.3 \\
\hline & $\dot{0}$ & 0.0424 & 174.9 & $7.7 \mathrm{e}-3$ & 155 & & 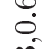 & 0.0023 & 2127.1 & $4.1 \mathrm{e}-4$ & 1379.4 \\
\hline & $\stackrel{?}{0}$ & 0.0425 & 122.7 & $2.9 \mathrm{e}-4$ & 243 & & $\dddot{m}$ & 0.0044 & 2543.1 & $5.5 \mathrm{e}-3$ & 964.9 \\
\hline & & 0.0419 & 126.4 & $3.5 \mathrm{e}-3$ & 260 & & & 0.0021 & 2479.3 & $7.9 \mathrm{e}-4$ & 1417.5 \\
\hline & $\overline{\boldsymbol{R}}$ & 0.663 & 0.427 & $3.14 \mathrm{e}-1$ & 0.38 & & $\overline{\boldsymbol{R}}$ & 0.447 & 0.563 & $4.02 \mathrm{e}-1$ & 0.535 \\
\hline
\end{tabular}

of the solutions obtained from the two algorithms; but as far as convergence and solution time were concerned, Simulated Annealing (SA) algorithm had higher performance than the Variable Neighborhood Search (VNS) algorithm. Future research on this topic may focus on customer service and arrival using other queuing models. Additionally, hierarchical models can be deployed to prioritize the requests and apply more restrictions on economic, competitive, or geographical conditions. 
Table 7. Results of $\bar{R}$ for examples 1-6 (continued).

\begin{tabular}{|c|c|c|c|c|c|c|c|c|c|c|c|}
\hline \multirow[b]{2}{*}{ 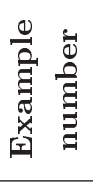 } & \multirow[b]{2}{*}{$\gamma_{j}$} & \multicolumn{2}{|c|}{ SA } & \multicolumn{2}{|c|}{ VNS } & \multirow[b]{2}{*}{ 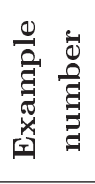 } & \multirow[b]{2}{*}{$\gamma_{j}$} & \multicolumn{2}{|c|}{ SA } & \multicolumn{2}{|c|}{ VNS } \\
\hline & & $\begin{array}{c}\text { LP } \\
\text { objective } \\
\text { value } \\
\end{array}$ & $\begin{array}{c}\text { LP } \\
\text { solution } \\
\text { time (s) }\end{array}$ & $\begin{array}{c}\text { LP } \\
\text { objective } \\
\text { value } \\
\end{array}$ & $\begin{array}{c}\text { LP } \\
\text { solution } \\
\text { time }(\mathrm{s})\end{array}$ & & & $\begin{array}{c}\text { LP } \\
\text { objective } \\
\text { value } \\
\end{array}$ & $\begin{array}{c}\text { LP } \\
\text { solution } \\
\text { time }(\mathrm{s}) \\
\end{array}$ & $\begin{array}{c}\text { LP } \\
\text { objective } \\
\text { value } \\
\end{array}$ & $\begin{array}{c}\text { LP } \\
\text { solution } \\
\text { time (s) }\end{array}$ \\
\hline \multirow{18}{*}{$\begin{array}{l}\text { } 8 \\
8 \\
11 \\
\text { है } \\
10\end{array}$} & \multirow{5}{*}{$\begin{array}{l}\Re \\
\vdots \\
-1 \\
0 \\
0 \\
0 \\
0\end{array}$} & 0.0051 & 2531 & $1.3 \mathrm{e}-5$ & 1958 & \multirow{18}{*}{0} & \multirow{5}{*}{$\begin{array}{l}\because \\
0 \\
-1 \\
0 \\
0 \\
0 \\
0\end{array}$} & 0.0083 & 3519.3 & $2.1 \mathrm{e}-4$ & 2351.1 \\
\hline & & 0.0086 & 2194 & $4.6 \mathrm{e}-5$ & 1620 & & & 0.0091 & 3873.4 & $9.5 \mathrm{e}-4$ & 2370.4 \\
\hline & & 0.0041 & 2223 & $4.9 \mathrm{e}-5$ & 1749 & & & 0.0042 & 3097.1 & $1.9 \mathrm{e}-4$ & 2528.6 \\
\hline & & 0.0055 & 2871 & $2.7 \mathrm{e}-5$ & 1737 & & & 0.0139 & 3627.6 & $8.7 \mathrm{e}-4$ & 2497.3 \\
\hline & & 0.0079 & 2196 & $4.4 \mathrm{e}-5$ & 1892 & & & 0.0071 & 3156.2 & $3.6 \mathrm{e}-4$ & 2616.7 \\
\hline & $\bar{R}$ & 0.431 & 0.308 & $6.33 \mathrm{e}-1$ & 0.50 & & $\bar{R}$ & 0.445 & 0.460 & $3.86 \mathrm{e}-1$ & 0.458 \\
\hline & \multirow{5}{*}{$\begin{array}{l}\stackrel{0}{0} \\
0 \\
0 \\
0 \\
0 \\
\stackrel{0}{0}\end{array}$} & 0.006 & 3610 & $2.4 \mathrm{e}-5$ & 1743 & & \multirow{5}{*}{$\begin{array}{l}\stackrel{0}{0} \\
\stackrel{0}{0} \\
\ddot{0} \\
\stackrel{-}{0}\end{array}$} & 0.081 & 3516.3 & $3.2 \mathrm{e}-5$ & 2961.6 \\
\hline & & 0.0074 & 3421 & $5.1 \mathrm{e}-5$ & 1515 & & & 0.0215 & 3254.7 & $4.1 \mathrm{e}-5$ & 3199.3 \\
\hline & & 0.0099 & 3930 & $2.3 \mathrm{e}-5$ & 1967 & & & 0.0098 & 3498.8 & $8.3 e-6$ & 3184.9 \\
\hline & & 0.0067 & 3492 & $3.7 \mathrm{e}-5$ & 1833 & & & 0.0229 & 3213.8 & $2.5 \mathrm{e}-5$ & 3024.5 \\
\hline & & 0.0059 & 3573 & $3.1 \mathrm{e}-5$ & 1792 & & & 0.0071 & 3921.7 & $2.7 \mathrm{e}-5$ & 3213.5 \\
\hline & $\bar{R}$ & 0.32 & 0.361 & $3.64 \mathrm{e}-1$ & 0.56 & & $\bar{R}$ & 0.555 & 0.377 & $5.61 \mathrm{e}-1$ & 0.61 \\
\hline & \multirow{5}{*}{$\begin{array}{l}-1 \\
0 \\
0 \\
0 \\
0 \\
0 \\
0 \\
0\end{array}$} & 0.0037 & 2219 & $8.2 \mathrm{e}-5$ & 1882 & & \multirow{5}{*}{$\begin{array}{l}-1 \\
0 \\
0 \\
0 \\
0 \\
0 \\
0\end{array}$} & 0.0194 & 3844.6 & $5.2 \mathrm{e}-6$ & 3487.5 \\
\hline & & 0.0029 & 2345 & $8.9 \mathrm{e}-5$ & 2330 & & & 0.0078 & 3768.1 & $3.7 \mathrm{e}-5$ & 3154.2 \\
\hline & & 0.0057 & 2109 & $5.6 \mathrm{e}-5$ & 1945 & & & 0.0116 & 3925.9 & $6.3 e-5$ & 3591.4 \\
\hline & & 0.0064 & 2367 & $7.1 \mathrm{e}-5$ & 1641 & & & 0.0085 & 3750.2 & $9.3 e-5$ & 3587.1 \\
\hline & & 0.0054 & 2480 & $7.7 \mathrm{e}-5$ & 2401 & & & 0.0071 & 3901.7 & $5.0 \mathrm{e}-6$ & 3347.9 \\
\hline & $\overline{\boldsymbol{R}}$ & 0.548 & 0.525 & $5.75 \mathrm{e}-1$ & 0.52 & & $\overline{\boldsymbol{R}}$ & 0.307 & 0.500 & $4.05 \mathrm{e}-1$ & 0.639 \\
\hline
\end{tabular}

\section{References}

1. Toregas, C., Swain, R., ReVelle, C. and Bergman, L. "The location of emergency service facilities", Operations Research, 19, pp. 1363-1373 (1971).

2. Shanthikumar, J.G. and Yao, D.D. "Optimal server allocation in a system of multi-server stations", Management Science, 33, pp. 1173-1191 (1978).

3. Hakimi, S.L. "On locating new facilities in a competitive environment", European Journal of Operational Research, 12, pp. 29-35 (1983).

4. ReVelle, C. and Hogan, K. "A reliability-constrained sitting model with local estimates of busy fractions", Environment and Planning B: Planning and Design, 15, pp. 143-152 (1988).

5. Marianov, V. and ReVelle, C. "The queuing probabilistic location set covering problem and some extensions", Socio-Economic Planning Sciences, 28, pp. 167-178 (1994).

6. Marianov, V., Serra, D. and Revelle, C. "Location of hubs in a competitive environment", European Journal of Operational Research, 114, pp. 363-371 (1999).

7. Marianov, V. and Serra, D. "Hierarchical locationallocation models for congested systems", European Journal of Operational Research, 135, pp. 195-208 (2000).

8. Marianov, V. and Serra, D. "Location-allocation of multiple-server service center with constrained queues or waiting time", Annals of Operations Research, 111, pp. 35-50 (2002).

9. Shavandi, H. and Mahlooji, H. "A fuzzy queuing location model with a genetic algorithm for congested systems", Applied Mathematics and Computation, 181, pp. 440-456 (2006).

10. Rajagopalan, H.K. and Saydam, C. "A minimum expected response model: Formulation, heuristic solution, and application", Socio-Economic Planning Sciences, 43, pp. 253-262 (2009).

11. Restrepo, M., Henderson, S.G. and Topaloglu, H. "Erlang loss models for the static deployment of ambulances", Health Care Management Science, 12, pp. 67-79 (2009).

12. Liu, Q. and $\mathrm{Xu}$, J. "A study on facility locationallocation problem in mixed environment of randomness and fuzziness", Journal of Intelligent Manufacturing, 3, pp. 389-398 (2011).

13. Chanta, S., Mayorga, M.E. and Mclay, L.A. "Improving emergency service in rural areas: a bi-objective covering location model for EMS systems", Annals of Operation Research, pp. 1027-1054 (2011).

14. Arnaout, J.P. "Ant colony optimization algorithm for the Euclidean location-allocation problem with unknown number of facilities", Journal of Intelligent Manufacturing, 24, pp. 45-54 (2011).

15. Drezner, T. and Drezner, Z. "The gravity multiple 
server location problem", Computers and Operations Research, 38, pp. 694-701 (2011).

16. Li, X., Zhao, Z., Zhu, X. and Wyatt, T. "Covering models and optimization techniques for emergency response facility location and planning: a review", Mathematic Meta Operations Research, 74, pp. 2811310 (2011).

17. Benneyan, J.C., Musdal, H., Ceyhan, M.E., Shiner, B. and Watts, B.V. "Specialty care single and multiperiod location allocation models within the veterans health administration", Socio-Economic Planning Sciences, 46, pp. 136-148 (2012).

18. Rahmati, S.H.A., Hajipour, V. and Akhavanniaki, S.T. "A soft computing pareto based meta heuristic algorithm for a multi objective multi server facility location problem", Applied Soft Computing, 13, pp. 1728-1740 (2013).

19. Mousavi, S.M. and Akhavan Niaki S.T. "Capacitated location allocation problem with stochastic location and fuzzy demand: A hybrid algorithm", Applied Mathematical Modeling, 37, pp. 5109-5119 (2013).

20. Adler, N., Hakkert, A.S., Kornbluth, J., Raviv, T. and Sher, M. "Location-allocation models for traffic police patrol vehicles on an interurban network", Ann. Oper. Res. (2013).

21. Goswami, V. "Analysis of discrete-time multi-server queue with balking", International Journal of Management Science and Engineering Management, 9(1), pp. 21-31 (2014).

22. Hansen, P. and Mladenovic, N. "Variable neighborhood search for the p-median", Location Science, 5, pp. 207-226 (1997).

23. Hajakbari, A. "Simulated annealing approach for solving stock cutting problem", IEEE International Conference on Systems Man and Cybernetics Conference Proceedings (1990).

24. Metropolis, N., Rosenbluth, A.W., Rosenbluth, M.N., Teller, A.H. and Teller, E. "Equation of state calculation by fast computing machines", Journal of Chem. Phys., 21, pp. 1087-1091 (1953).

25. Kirkpatrick, S., Gellat, C.D. and Vecchi, M.P. "Optimization by simulated annealing", Science, 220, pp. 671-680 (1983).

26. Deb, S.K. "Solution of facility layout problems with pickup/drop-off locations using random search techniques", International Journal of Production Research (2005).

\section{Biographies}

Maryam Ghobadi is a PhD student in the Department of Industrial Engineering at Kordestan University of Iran. She received her MSc degree in Industrial Engineering from Alzahra University in 2013. Her current research interests include location and supply chain management.

Mehdi Seifbarghy is Professor in the Department of Industrial Engineering at Alzahra University of Iran and presently serves as the Vice President of Academic Affairs. In teaching, he has been focusing on location and facility layout problems and supply chain management. In research, his current interests include location and supply chain management. Dr. Seifbarghy received his $\mathrm{PhD}$ degree in Industrial Engineering from Sharif University of Technology, Tehran, Iran.

Reza Tavakkoli-Moghaddam is Professor of Industrial Engineering at University of Tehran, Iran. He obtained his PhD in Industrial Engineering from the Swinburne University of Technology in Melbourne (1998). He is an Associate Member at Academy of Sciences in Iran and serves as Editorial Board Member of the International Journal of Engineering and Iranian Journal of Operations Research. He was the recipient of the 2009 and 2011 Distinguished Researcher Awards and the 2010 Distinguished Applied Research Award at University of Tehran, Iran. He was selected as National Iranian Distinguished Researcher in 2008 and 2010 in Iran. Professor Tavakkoli-Moghaddam has published 4 books, 15 book chapters, and more than 500 papers in reputable academic journals and conferences.

Davar Pishva is a Professor in ICT at the College of Asia Pacific Studies, Ritsumeikan Asia Pacific University (APU) Japan. In teaching, he has been focusing on information security, technology management, VBA for modelers, structured decision making and carries out his lectures in an applied manner. In research, his current interests include biometrics; e-learning, environmentally sound and ICT enhanced technologies. Dr. Pishva received his PhD degree in System Engineering from Mie University, Japan. He is a Senior Member of IEEE, a member of IEICE (Institute of Electronics Information \& Communication Engineers), and University \& College Management Association. 